\title{
O Direito à Saúde como um direito humano em Moçambique
}

\author{
The Right to Health as a Human Right in Mozambique \\ El derecho a la salud como un derecho humano en Mozambique
}

Orquídea Massarongo-Jona ${ }^{1}$

\begin{abstract}
RESUMO: O presente artigo analisa a questão do acesso e disponibilidades dos serviços de saúde e medicamentos essências como componentes do direito à saúde, concentrando-se nas obrigações do Estado moçambicano resultantes dos instrumentos internacionais de direitos humanos em particular na vertente dos direitos económicos, socais e culturais, tendo em conta a vulnerabilidade da população moçambicana para doenças endémicas, em particular a malária. A abordagem considera a recente avaliação de desempenho de Moçambique relativa aos direitos humanos no que concerne ao direito à saúde através do Mecanismo de Revisão Periódica Universal. O artigo foca nos 4 critérios de abordagem dos Direitos Humanos fixados pela ONU e que constituem a base da avaliação da situação do direito a saúde em Moçambique: Disponibilidade, Acessibilidade, Aceitabilidade e Qualidade (DAAQ) e como o quadro legal moçambicano vigente responde as obrigações internacionais de protecção do direito à saúde.
\end{abstract}

Palavras-chave: Direito à Saúde. Direitos Humanos. Discriminação. Acessibilidade.

ABSTRACT: This article analyzes the issue of access and availability of health services and essential medicines as components of the right to health, focusing on the obligations of the Mozambican State resulting from international human rights instruments, in particular in economic, social rights Taking into account the vulnerability of the Mozambican population to endemic diseases, particularly malaria. The approach considers the recent assessment of Mozambique's human rights performance about the right to health through the Universal Periodic Review Mechanism. The article focuses on the four criteria for approaching human rights established by the UN and which form the basis for assessing the situation of the right to health in Mozambique: Availability, Accessibility, Acceptability and Quality (DAQA) and how the current Mozambican legal framework responds to obligations Protection of the right to health.

Keywords: Right to Health. Human Rights. Discrimination. Accessibility

\footnotetext{
${ }^{1}$ Docente na Faculdade de Direito da Universidade Eduardo Mondlane (UEM). Estudante em nível de Doutoramento em Direitos Humanos na Universidade de Ghent - Bélgica na área de Saúde Sexual e Reprodutiva. Possui Mestrado em Direito do Comércio Internacional, Universidade de Stellenbosch, África do Sul (2003). É Coordenadora do Projecto de Direitos Humanos e do Mestrado em Direitos Humanos no âmbito do Programa Desafio (Cooperação interuniversitária entre a UEM e Universidades Flamengas). Pesquisadora com interesse na área Direito da Saúde, Direitos humanos das mulheres e Direitos Humanos e Negócios. Membro da Comissão Nacional de Bioética para Saúde e Vice-Presidente do Conselho Nacional da Ordem dos Advogados de Moçambique. Membro da Rede de Universidades do Programa de Direitos Humanos e Democratização em África em parceria com a Universidade de Pretória (África do Sul). É Coordenadora (Organizadora) da Revista de Direitos Humanos editada pelo Centro de Direitos Humanos da UEM.Email: orquidiamz@yahoo.com.br
} 
RESUMEN: En este artículo se examina la cuestión del acceso y disponibilidad de servicios de salud y medicamentos esencias como componentes del derecho a la salud, centrándose en las obligaciones del Estado de Mozambique resultante de los derechos humanos internacionales, en particular en el aspecto de los derechos económicos, sociais y cultural, teniendo en cuenta la vulnerabilidad de la población de Mozambique a las enfermedades endémicas, especialmente la malaria. El enfoque tiene en cuenta la reciente evaluación del desempeño de Mozambique en materia de derechos humanos en relación con el derecho a la salud a través del Mecanismo de Examen Periódico Universal. El artículo se centra en 4 criterios de aproximación de los derechos humanos establecidos por la ONU y que forman la base de la evaluación de la situación de los derechos de salud en Mozambique: disponibilidad, accesibilidad, aceptabilidad y calidad (DAAQ) y cómo el marco legal vigente en Mozambique responde obligaciones la protección internacional del derecho a la salud.

Palabras-Ilave: Derecho a la Salud. Derechos Humanos. La discriminación. Accesibilidad

\section{Introdução}

A Declaração Universal dos Direitos Humanos (DUDH) determina que "toda a pessoa tem direito a um nível de vida suficiente para lhe assegurar [a si] e à sua família a saúde e o bem estar, principalmente quanto à alimentação, ao vestuário, à assistência médica e ainda quanto aos serviços sociais necessários (...)”2 portanto é indiscutível que a saúde é um direito de todos (1), qualquer pessoa tem o direito de obter atendimento e acesso aos serviços básicos de saúde e medicamentos considerados essenciais tal como definidos pela OMS (Organização Mundial da Saúde) (2), (3).

O Direito da Saúde corresponde a um conjunto de normas de direito, privado e público, com objectivo principal de promoção da saúde humana, quer considerada na perspectiva da prestação de cuidados individuais, quer enquanto bem de uma comunidade.

A Constituição da República de Moçambique (CRM 2004), no capítulo V referente aos direitos sociais garante, no artigo 89, a todo cidadão o direito a saúde. Estes fazem parte do chamado grupo de direitos fundamentais de segunda geração ${ }^{3}$, "os direitos sociais, culturais e económicos", que estão ligados intimamente a direitos de prestações sociais do

\footnotetext{
${ }^{2}$ Vide Art. 25 da Declaração Universal dos Direitos Humanos (1948).

${ }^{3}$ Os direitos Fundamentais são classificados em gerações, sendo os da primeira geração correspondentes aos direitos clássicos civis e políticos; os direitos sociais, económicos e culturais correspondem a segunda geração; os de fraternidade e solidariedade em que se inclui à paz, meio ambiente, património, etc correspondem a terceira geração e por último os da quarta geração que se referem a institucionalização do Estado Social e compreende o direito à democracia, à informação e ao pluralismo.
} 
Estado perante o indivíduo, bem como a assistência social, educação, saúde, cultura e trabalho.

Para a nossa a reflexão, julgamos ser relevante fazer referencia a classificação tripartida do direito da saúde, posto que a nossa abordagem irá incidir numa delas, o acesso aos serviços de saúde. Com base nesta classificação direito da saúde divide-se em três partes, nomeadamente: (i) Direito da Saúde Pública que regula a intervenção do Estado na protecção e promoção da saúde pública; (ii) Direito da Medicina que regula a relação médico paciente e por último (iii) Direito das Prestações de Saúde que regula o acesso do cidadão às prestações de saúde.

O presente artigo irá debruçar-se resumidamente sobre o acesso aos serviços de saúde e medicamentos essências como componentes do direito à saúde, concentrando-se nas obrigações do Estado moçambicano resultantes dos instrumentos internacionais de direitos humanos em particular na vertente dos direitos económicos, socais e culturais, tendo em conta a vulnerabilidade da população moçambicana para doenças endémicas, em particular a malária. A abordagem tomará em conta a recente avaliação de Moçambique no que concerne ao direito à saúde através do Mecanismo de Revisão Periódica Universal (MRPU).(4)

\section{Direito à Saúde como Direito Humano: Visão Geral}

O Direito à saúde é parte de um conjunto de direitos chamados de direitos sociais(5) considerado como um dos direitos mais difíceis de proteger pois para a sua consecução implica acções efectivas e proactivas dos Estados para a realização do direito(6). Os direitos sociais foram instituídos para que as condições razoáveis de vida em sociedade pudessem ser gozadas por todos.

Então o que engloba o direito da saúde? Que conjunto de direitos são esses e em que consistem? Como ponto de partida, comecemos pela conceptualização do direito à saúde, a partir da definição de saúde apresentada pela Organização Mundial da Saúde (OMS): a saúde "é um estado de completo bem-estar físico, mental e social, e não consiste apenas na ausência de doença ou de enfermidade." (7)

O direito à saúde engloba o conjunto de normas jurídicas que estabelecem os direitos e obrigações do Estado, colectividades e que regulam as relações entre o Estado e as colectividades (5) 
O direito à saúde, nos termos do Pacto Internacional dos Direitos Econômicos, Sociais e Culturais (PIDESC), consiste no direito ao tratamento médico e serviços preventivos de saúde para que os cidadãos possam alcançar o mais alto nível de saúde física e mental.

$\dot{E}$ ponto assente, que o direito à saúde deve ser entendido como o direito de beneficiar ou gozar de um conjunto de facilidades, bens, serviços e condições necessárias para a realização ao mais alto nível possível de atingir, estando aqui realçado a questão da disponibilidade e acesso aos serviços de saúde. O mesmo acontece com o acesso a medicamentos essenciais que constitui um elemento fundamental do direito à saúde.

O direito à saúde, inclui também o acesso ao tratamento médico em caso de doença, bem como a prevenção, tratamento e controle de enfermidades são atributos centrais do direito a desfrutar do mais elevado nível possível de saúde, atributos que dependem do acesso a medicamentos e da cobertura universal dos serviços de saúde (8) Falar de direito à saúde é também referir-se ao acesso e cobertura universal (5)dos serviços de saúde, em qualidade e quantidade para toda a população.

Do acima exposto pode-se depreender que definir o conteúdo do direito à saúde é um desafio, mas que não impediu que este direito fosse reconhecido (9), não obstante as dificuldades de implementação na práctica. O Comité dos Direitos Económicos, Sociais e Culturais (CDESC), órgão responsável pela implementação e monitoria do PIDESC instituiu o Comentário Geral número 14 que define o conteúdo e alcance do direito a saúde e as medidas que os Estados devem adoptar para a realização deste direito (9).

\section{Contexto social e económico de Moçambique relacionado com a Saúde}

Moçambique é considerado como um dos países mais pobres e menos desenvolvidos do mundo, ${ }^{4}$ com uma população estimada de 28.943.604 habitantes (em setembro de 2016). ${ }^{5}$ Da análise efectuada nos documentos oficiais e relatórios disponíveis do Ministério da Saúde de Moçambique, preocupam a saúde pública em Moçambique a malária,

\footnotetext{
${ }^{4}$ Segundo dados divulgados em 2010, no período de 2008/2009, de 21,5 milhões de pessoas em Moçambique, $55 \%$ da população vivem abaixo da linha de pobreza com cerca de meio dólar americano por dia. Vide Ministério de Planificação e Desenvolvimento (MPD), Direcção Nacional de Estudos e Análises de Politicas, (2010) Terceira

Avaliação Nacional sobre Pobreza e Bem-Estar em Moçambique, p. xiii; MISAU (2013) Plano Estratégico da Sector da Saúde 2014-2019, Ministério da Saúde (PDC), p. 12.

${ }^{5}$ http://countrymeters.info/pt/mozambique
} 
HIV/SIDA, tuberculose, diarreia, infecções respiratórias, sarampo e cólera, com destaque para a malária, que é endémica em todo o país e constitui um dos principais desafios para a saúde pública e para o desenvolvimento sustentável do país. ${ }^{6}$

Em Moçambique a malária é a principal causa de problemas de saúde e principal causa de morte. Constatou-se que $45 \%$ das admissões em consultas externas e $56 \%$ de internamento em enfermarias de pediatria se devem à Malária. Segundo o último inquérito demográfico de saúde (IDS 2011) a prevalência da malária em crianças é de 35,1\% (10)

$\mathrm{O}$ acesso aos cuidados em saúde é muito baixo no país e estima-se que cerca de $50 \%$ da população vive a mais de 20 quilômetros da unidade de saúde mais próxima e 62,4\% sem acesso a serviços de saúde (o país ocupa a posição 183 entre 191 países analisados quanto à densidade de médicos). A percentagem da população com acesso a uma unidade sanitária a menos de 45 minutos a pé aumentou de $55 \%$ para $65 \%$ entre 2002-2003 e 2008-2009, com maiores índices para as zonas rurais (especialmente no norte do país) comparado com as zonas urbanas, onde se observa uma redução da população com acesso a uma unidade sanitária a menos de 45 minutos de distância, indicando a necessidade de expansão rápida das zonas urbanas. ${ }^{7}$

Os dados estatísticos e relatórios de organizações não-governamentais revelam que a população moçambicana em particular da zona rural, que constitui a maioria, para ter acesso aos serviços de saúde deve percorrer longas distâncias, pois não tem opção de transporte público e mesmo depois de percorrer a pé, fá-lo sem garantias de atendimento e tratamento com qualidade e dignidade humana (11), dadas as enchentes e condições de atendimento no território.

Em Moçambique, a assistência é feita por meio do Sistema Nacional de Saúde (SNS) que beneficia todo o povo moçambicano tal como disposto na $l \mathrm{e}^{8}$ e $\mathrm{o}$ Estado

\footnotetext{
${ }^{6}$ Dados de cinco anos, referente ao período de 2009 a 2013 do sistema de vigilância epidemiológica (Boletim Epidemiológico Semanal, BES do Ministério da Saúde -MISAU), a malária conta com uma média de 5,8 milhões de casos diagnosticados clinicamente por ano, sendo a principal razão de consulta externa (45\%) e de internamento no serviço de pediatria (56\%) e com alta taxa de mortalidade (variação de $1.8 \%$ a $9.9 \%$, dependendo do nível da unidade sanitária). Dados mais recentes, revelam que só primeiro semestre de 2014, foram registados 3.297 .386 casos de malária e 1.937 óbitos em todo o País. Para além do impacto directo na saúde, existe um peso socioeconómico enorme nas comunidades e no país em geral, particularmente para os segmentos populacionais mais pobres e vulneráveis.

<http://www.aho.afro.who.int/profiles information/index.php/Mozambique:Introduction to Country Context/pt 8 Art. 116 no1 da CRM 2004 e art. 1 da Lei2/77, de 19 de Janeiro referente ao Lei do Sistema Nacional de Saúde (SNS), que estabelece que são gratuitas as acções sanitárias de carácter profiláctico [sem no
} 


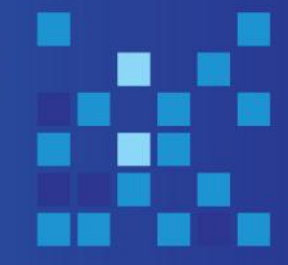

responsabiliza-se pelo acesso aos serviços de saúde para todos os moçambicanos. ${ }^{9}$, no entanto o alcance do Serviço Nacional de Saúde é reduzido nas zonas rurais comparativamente a zona urbana. (12)

O Sistema Nacional de Saúde funciona na base de gratuidade para um conjunto de serviços de saúde e uma taxa simbólica para internamento e tratamento ambulatório, que pelo nível de pobreza, falta de fontes de rendimento estáveis e conjugado com os níveis de desemprego no país, os valores instituídos pelas taxas nos serviços de saúde, por mais baixos que sejam afectam o acesso aos serviços de saúde, na medida que que essa taxa é acrescida a outros custos tais como medicamentos, consulta, transporte e alimentação.

\section{Quadro Normativo do Direito a saúde}

Pelo facto dos direitos humanos serem direitos interdependentes e interrelacionados, no sentido de que os direitos humanos não funcionam isoladamente e que todos os direitos devem ser reconhecidos. O direito humano à saúde, não é um direito recente, foi reconhecido em 1948 com a Declaração Universal (DUDH) que assegura ao ser humano saúde e bem-estar com relação a vários factores dos quais a assistência médica, ${ }^{10} \mathrm{e}$ reafirmado em 1966 com o Pacto Internacional sobre os Direitos Económicos, Sociais e Culturais (PIDESC), ${ }^{11}$ o qual traduz os princípios da Declaração Universal de Direitos Humanos em instrumento legalmente vinculativo, ${ }^{12}(13)$ apenas aos Estados parte do PIDESC, ${ }^{13}$ reconhecendo o 'direito de todas as pessoas de gozar do melhor estado de saúde física e mental.'

entanto indicar na lei as situações que se enquadram nesse carácter], no art. 2 determina que "todo o cidadão tem direito à assistência médica e medicamentosa gratuita quando em regime de internamento" e no art. 6 fixa que a gratuidade no tratamento ambulatório é estabelecida para os medicamentos considerados básicos. Vide art. ${ }^{\circ} 1$ nำ \& nำ da Lei 4/87, de 19 de Janeiro, que trouxe algumas alterações a Lei do Sistema

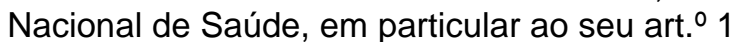

${ }^{9}$ Art. ${ }^{\circ} 49$ da CRM.

${ }^{10}$ Art. 025 (1) da DUDH.

${ }_{11}^{11}$ Art. -12 (1) do PIDESC. O PIDESC foi adoptado em 1966 e entrou em vigor em 1976.

12 O PIDESC corresponde a transformação das disposições da Declaração Universal (DUDH), juntamente com o PIDCP (Pacto Internacional sobre os Direitos Civis e Políticos), em obrigações com forca legal, 'which are the bedrock of the international normative regime for human rights.

${ }_{13}$ Moçambique não é signatário do PIDESC, contudo como membro da ONU está obrigado a não tomar nenhuma medida contra a finalidade e regras plasmadas no Pacto. 


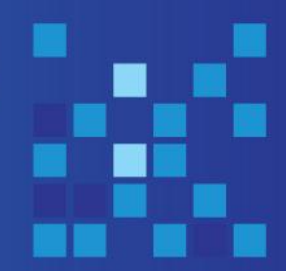

Uma característica ou aspecto a destacar do PIDESC é o princípio da "implementação progressiva" ${ }^{14}$ o que implica que a realização do direito não é imediata uma vez que exige meios e recursos, impondo um conjunto de obrigações essenciais mínimos aos Estados, sendo uma das obrigações mínimas a de "garantir o acesso as unidades hospitalares e serviços de forma não discriminatória, em particular para os grupos vulneráveis." ${ }^{15}$ Contudo, este princípio não deve constituir motivo ou justificação para o Estado, em particular os países menos desenvolvidos e com menos recursos, como Moçambique, para não realizar o direito humano à saúde, posto que o Estado impossibilitado por falta de recursos deve justificar e demonstrar que usou de todos os recursos e meios disponíveis com vista a satisfação do direito. ${ }^{16}$

Em nível regional africano, o quadro normativo do direito a saúde é definido a partir do artigo 16 da Carta Africana dos Direitos Humanos e dos Povos (CADHP) da União Africana, o qual determina que "toda a pessoa tem direito ao gozo do melhor estado de saúde física e mental que for capaz de atingir" ${ }^{17}$ Com este instrumento regional os Estados Africanos obrigam-se a tomar as "medidas necessárias para proteger a saúde das suas populações e para Ihes assegurar assistência médica em caso de doença." ${ }^{18}$

\section{Obrigações do Estado Moçambicano em relação ao Direito à saúde}

Para garantir que as pessoas em sociedade possam desfrutar do mais elevado nível possível de saúde, os direitos humanos impõem aos Estados três níveis de obrigações, ${ }^{19}$ nomeadamente obrigação de respeitar, proteger e cumprir ou implementar o direito à saúde. A obrigação de respeitar o direito humano à saúde obriga o Estado a não interferir ou negar o gozo do direito; o direito de proteger exige que o Estado deve prevenir e

\footnotetext{
${ }^{14}$ Vide art. ${ }^{0} 2.1$ do PIDESC "Cada um dos Estados (...) compromete-se a agi $\mathrm{Vr}$ (...) no máximo dos seus recursos disponíveis, de modo a assegurar progressivamente o pleno exercício dos direitos reconhecidos no presente Pacto por todos os meios apropriados, incluindo em particular por meio de medidas legislativas."

${ }^{15}$ CDESC Comentário Geral No. 14 Paragrafo 43, alínea a).

${ }^{16} \mathrm{O}$ CDESC no seu Comentário Geral No. 14 Paragrafo 47 distingue quando se deve considerar que o Estado está 'impossibilitado' por falta de recursos ou por 'falta de vontade' não realizou o direito a saúde, o que no ultimo caso considera-se que esta em violação das obrigações nos termos do art. ${ }^{\circ} 12$ do PIDESC.

${ }^{17}$ Art. 16 (1) CADHP.

${ }^{18}$ Art. 16 (2) CADHP. Dos 56 países membros da União Africana, apenas 3 países (Botswana, Egipto e a Tunísia) não são signatários da Carta.

${ }^{19}$ CDESC Comentário Geral No. 14 Paragrafo 33.
} 
assegurar que terceiros não impeçam a realização do direito à saúde ${ }^{20}$ e o dever de cumprir requer que o Estado seja proactivo na garantia do acesso aos cuidados de saúde, providenciando por exemplo um número suficiente de unidades hospitalares e centros de saúde, em particular para as zonas rurais que mais necessitam.

No que concerne a obrigação de implementação do direito à saúde, as obrigações do Estado com relação ao direito à saúde, consistem em: ${ }^{21}$

- Reconhecimento adequado do direito à saúde na legislação e políticas nacionais;

- Acesso em termos de igualdade e sem discriminação, a todos factores determinantes de saúde, tais como alimentação, água potável, saneamento básico, habitação adequada;

- Providenciar nas Unidades Hospitalares Públicas serviços de saúde sexual e reprodutiva;

- Formar adequadamente do pessoal de saúde (médicos e outros);

- Providenciar número suficiente de hospitais, clínicas e centros de saúde e outras unidades de saúde relacionadas;

- Estabelecer instituições de aconselhamento e serviços de saúde mental.

De acordo com os princípios de Limburgo, ${ }^{22}$ os Estados devem tomar medidas legislativas, administrativas, judiciais, económicas, sociais e educacionais e se necessário criar espaço para que medidas judiciais para reparação ou restituição do direito violado. Com relação ao direito à saúde, o Comité dos Direitos Económicos e Sociais determina que os Estados têm no mínimo que cumprir as seguintes obrigações essenciais: (i) garantir o acesso a unidades hospitalares (centros de saúde ou outros), bens e serviços; (ii) Providenciar medicamentos essenciais tal como definidos pela OMS e (iii) garantir uma distribuição equitativa das unidades, bens e serviços de saúde. ${ }^{23}$

\footnotetext{
${ }^{20}$ Esta obrigação pode ser cumprida através de medidas legislativas e monitoria dos serviços de saúde em caso de terceirização ou privatização dos serviços de saúde.

${ }^{21}$ CDESC Comentário Geral No. 14 Parágrafo 37.

22 São princípios elaborados em 1986 pela Comissão Internacional de Juristas (http://www.icj.org ) que ilustram as obrigações dos Estados relacionados com os direitos económicos, sociais e culturais resultantes do PIDESC. Disponível em $<$ https://www.escr-net.org/resources/limburg-principles-implementationinternational-covenant-economic-social-and-cultural >

${ }^{23}$ CDESC Comentário Geral No. 14 paragrafo 43, alínea a), d) e c).
} 


\section{Alguns aspectos do Direito à Saúde como direito humano em Moçambique}

Como afirmado, o Estado moçambicano não ratificou o PIDESC, contudo dedicou um capítulo na Constituição da República que reflecte os princípios e normas do PIDESC, ${ }^{24} \mathrm{e}$ ainda, como membro da ONU, para além de estar obrigado a não agir em sentido diverso ou contra os demais instrumentos, acaba indirectamente por ficar abrangido pelos padrões, princípios e quadro normativo daquele organismo no que concerne aos direitos humanos, uma vez que a funciona de forma integrada com as suas várias agencias e com a OMS em particular, do qual o país é membro. A ONU fixou em 2003 um entendimento comum sobre a abordagem baseada nos direitos humanos, ${ }^{25}$ com vista ao desenvolvimento e um conjunto de ferramentas que constituem a base do direito à saúde que gira em torno de 4 critérios: o da disponibilidade, acessibilidade, aceitabilidade e qualidade (DAAQ).

Pelo critério da disponibilidade considera-se que Estado cumpre com o direito à saúde quando providencia serviços de saúde pública, instalações, bens e serviços de saúde disponíveis e funcionais em quantidade suficiente, com profissional qualificado e com medicamentos considerados essenciais conforme os padrões e directivas da OMS. ${ }^{26}$ Com relação a este critério, não é necessário uma lupa, para avaliar a situação do direito a saúde em Moçambique, porque existem vários relatórios ${ }^{27}$ e estudos que revelam que o país ainda não tem unidades hospitalares e sanitárias suficientes para a população. As unidades existentes não dão resposta às necessidades da população, faltam médicos e pessoal suficiente, quantidade insuficiente e funcionamento deficiente dos laboratórios quer por falta de técnicos e quer por falta de instrumentos de trabalho, escassez de medicamentos essencias e em alguns casos os custos associados.

Com relação ao critério da acessibilidade, será analisado nas suas quatro dimensões, nomeadamente discriminação, acessibilidade física, económica e acesso à informação. As

\footnotetext{
${ }^{24}$ Vide Capitulo V, Direitos e Deveres Económicos, Sociais e Culturais; Vide Relatório de Moçambique (2016) no âmbito do Mecanismo de Revisão Periódica Universal (MRPU) no período de 2011 a 2016 p.3. Disponível em <http://www.upr-info.org/sites/default/files/document/mozambique/session 24 january 2016/unct mozambique upr24 moz e main.pdf>

${ }^{25}$ Reafirmada pelo Secretário Geral da ONU em 2008, através da Policy Decision 2008/18 que recomendou a OMS e a as Agencias da ONU que trabalham na área da saúde para continuarem a reforçar a sua capacidade para integrar a abordagem baseada nos direitos humanos e advogar para os direitos humanos relacionados com a saúde. http://www.ohchr.org/Documents/lssues/ESCR/Health/HRBA HealthlnformationSheet.pdf

${ }^{26}$ CDESC Comentário Geral número 14 paragrafo 12, alínea a).

${ }^{27}$ Relatório de Moçambique no âmbito do MRPU (2016), p. 9.
} 
instalações, bens e serviços de saúde devem ser acessíveis para todos no país sem discriminação, em particular para as populações mais vulneráveis e marginalizadas. Para a dimensão da acessibilidade física as instalações, bens e serviços de saúde devem estar a uma distância de acesso seguro para todos os segmentos populacionais e com acesso para pessoas com deficiência. Com relação a acessibilidade económica, os serviços devem ser economicamente acessíveis para todos incluindo os grupos mais empobrecidos (8). O acesso às instalações e serviços de saúde são em princípio gratuitos em Moçambique. ${ }^{28}$.

E por último, a dimensão do acesso a informação que implica o direito de procurar, receber e transmitir informação relacionada com a saúde. O Ministério da Saúde-MISAU tem feito campanhas visíveis sobre os problemas de saúde, o que é preciso aferir e se essa informação é devidamente recebida e transmitida tendo em conta as barreiras culturais e linguísticas e bem como tendo em conta a relação médico paciente e pessoal de saúde e se estes não constituem a principal barreira na transmissão da informação. ${ }^{29}$

Para o critério da aceitabilidade, que se refere ao respeito pela ética médica, requisitos culturalmente apropriados, sensíveis ao género e às condições do ciclo da vida ${ }^{30}$ podemos realçar que as condições de atendimento, as enchentes e as longas filas de espera, associado ao cansaço do pessoal de saúde, muitas vezes tem criado dificuldades e constrangimento que resultam no mau atendimento, algumas vezes involuntário e colocando em risco a confidencialidade dos pacientes. ${ }^{31}$

Pelo critério da qualidade, os serviços e bens de saúde devem ser científicos e medicamente apropriados e de boa qualidade, ${ }^{32}$ constituíndo obrigação do Estado o estabelecimento de um sistema regulatório para verificar a segurança e qualidade dos serviços e bens, tendo em conta o risco de falsificação.

A recente avaliação de Moçambique no último relatório do Mecanismo de Revisão Periódica Universal da ONU, o acesso da população aos serviços e profissionais de saúde é limitado, com apenas $36 \%$ da população com esse direito garantido, mesmo assim em

\footnotetext{
${ }^{28}$ Sujeitos a uma taxa mínima, incluindo serviços de internamento hospitalar nos termos da Lei 2/77 de 27 de setembro de 1977. (Lei que regula a criação de taxas de internamento hospitalar).

${ }^{29}$ CDESC Comentário Geral No. 14 paragrafo 12, alínea b).

${ }^{30}$ CDESC Comentário Geral No. 14 paragrafo 12, alínea c).

${ }^{31}$ Não constitui novidade e existem relatos de pessoas que foram atendidas em condições que não permitem o respeito pela confidencialidade, em particular doentes de HIV/SIDA.

${ }^{32}$ CDESC Comentário Geral Número. 14 Parágrafo 12, alínea d).
} 
um espaço de 45 minutos a pé de distância e a ratio de profissionais de saúde/população é de 5.6 médicos para 100.000 habitantes. ${ }^{33}$ Esses dados dizem muito sobre a situação e do caminho que se tem que percorrer para a realização do direito á saúde em Moçambique.

\section{Conclusão}

O direito à saúde em Moçambique é garantido, pelo menos em termos formais pelo legislador moçambicano através da Constituição, leis e dos vários instrumentos internacionais que abordam o direito à saúde e ratificados por Moçambique. Contudo, a perspectiva de protecção desse direito, no componente de acesso aos serviços de saúde e medicamentos essenciais não é o mais adequado, o que choca com a característica da universalidade do direito à saúde como direito humano.

O Estado moçambicano ainda está longe de assegurar a plenitude de acesso e cobertura universal aos serviços de saúde de forma equitativa em todo o território nacional, beneficiando as zonas urbanas e as populações residentes das zonas rurais, o que vale dizer que para essas populações não se pode falar em direito à saúde.

A falta de acesso e cobertura universal dos serviços de saúde constitui um impedimento na realização do direito à saúde, do qual destaca-se as longas distâncias percorridas pelos doentes, a baixa disponibilidade de infraestrutura hospitalar e pessoal de saúde qualificado, a pouca disponibilidade de medicamentos, qualidade dos serviços prestados e recursos humanos capacitados.

Não obstante este cenário pouco colorido, Moçambique tem tomado medidas e registado melhorias, embora não na velocidade desejada ou desejável. Porém, por se tratar de um direito de realização progressiva e dependente de recursos financeiros de que Estado dispõe não se poderá falar da violação do direito à saúde, pois o Estado só está obrigado até ao limite dos seus recursos desde que seja respeitado com relação ao direito à saúde o leque de obrigações mínimas essências relativas aos direitos económicos sociais e culturais.

\footnotetext{
${ }^{33}$ Relatório de Moçambique (2016) no âmbito do Mecanismo de Revisão Periódica Universal (MRPU), p. 9.
} 


\section{Referências}

1 Ibrahim, F. Curso de Direito Previdenciário, Rio de Janeiro: Impetus, 2009

2 OMS, Folha Informativa Geral sobre o Direito à Saúde. Diploma Ministerial 54/2010, de 23 de Março. Disponível em http://www.who.int/selection_medicines/list/en/ [Acesso: 25 set 2016].

3 Humenhuk, $\mathrm{H}$. $\mathrm{O}$ direito à saúde no Brasil e a teoria dos direitos fundamentais. Jus Navigandi, Teresina, 9 (22):227, Disponível em: <http://jus.uol.com.br/revista/texto/4839>, Acesso em 18 set 2016.

4 MOZAMBIQUE. Ministério da Saúde-MISAU. Plano Estratégico da Malária 2012-2016, Ministério da Saúde (PNCM), Disponível em

http://www.misau.gov.mz/pt/programas/malaria/relatorio_de_malaria_2007. Acesso em 23 nov 2010.

5 Pessoa, E. A Constituição Federal e os Direitos Sociais Básicos ao Cidadão Brasileiro. In: Âmbito Jurídico, Rio Grande, XIV, n. 89, jun 2011. Disponível em: <http://www.ambitojuridico.com.br/site/index.php?n_link=revista_artigos_leitura\&artigo_id=9623>. Acesso em 14 set 2016.

6 Moura Gomes, J. (2009). Acesso a medicamentos como direito humano. Tese de doutoramento defendida junto a Universidade de São Paulo-USP, 2009.

7 Mitano, F., Ventura, CAA., Lima, MCRAD., Balegamire, JB., Palha, PF. Direito à Saúde:(in) congruência entre o arcabouço jurídico e o sistema de saúde. Revista latinoamericana de enfermagem, 24(1):1-7, 2016.

8 OMS. Preâmbulo da Constituição da OMS, Disponível em <http://apps.who.int/gb/bd/PDF/bd47/EN/constitution-en.pdf?ua=1> Acesso: 16 set 2016.

9 Hunt, P.; Khosla, R. Acesso a medicamentos como um direito humano. Sur, Rev. int. direitos human. 5(8), 2008.

10 Kinney, E. D. The International Human Right to Health: What Does This Mean for Our Nation and World?. Indiana Law Review, 34: 1457, 2001.

11 Arroz, J (2016), Aumento dos Casos de Malária em Moçambique 2014: epidemia ou novo padrão de endemicidade?. Rev. de Saúde Pública (RSP), 50(5):2, 2016.

12 MOZAMBIQUE. Ministério da Saúde-MISAU. Programa Nacional de Controle da Malária: Plano Estratégico da Malária 2012-2016. Disponível em:

http://www.misau.gov.mz/pt/programas/malaria. Acesso: 25 set 2016

13 Meneses, MPG. Medicina tradicional, biodiversidade e conhecimentos rivais em Moçambique. Disponível em < http://www.ces.uc.pt/publicacoes/oficina/150/150.pdf > Acesso em 28 set 2016 
14 Alston, P.; Goodman, R., International Human Rights: The Successor to International Human Rights in Context: Law, Politics and Morals. England: Oxford University Press, 2013.

Recebido em: 19/12/2016

Aprovado em: 29/12/2016

Como citar este artigo:

Jona-Massarongo O. O Direito à Saúde como um direito humano em Moçambique. Revista Cadernos IberoAmericanos de Direito Sanitário. 2016 dez., 5 supl. 1:152-164. 